\title{
X-Ray Magnetic Circular Dichroism Studies on $\mathrm{CeNi}_{4} \mathrm{~B}$
}

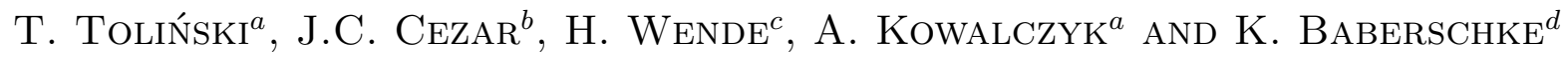 \\ ${ }^{a}$ Institute of Molecular Physics, Polish Academy of Sciences \\ M. Smoluchowskiego 17, 60-179 Poznań, Poland \\ ${ }^{b}$ E.S.R.F., 6 rue Jules Horowitz, 38043 Grenoble Cedex, France \\ ${ }^{c}$ Universität Duisburg-Essen Fachbereich Physik \\ Lotharstr. 1, 47048 Duisburg, Germany \\ ${ }^{d}$ Institut für Experimentalphysik, Freie Universität Berlin \\ Arnimallee 14, D-14195 Berlin-Dahlem, Germany
}

\begin{abstract}
The $\mathrm{CeNi}_{4} \mathrm{~B}$ compound is a mixed-valence system. It is a paramagnet with $\mu_{\text {eff }}=0.52 \mu_{\mathrm{B}} /$ f.u. and the paramagnetic Curie temperature $\theta=-10.7 \mathrm{~K}$. In the present research we complete our previous magnetic susceptibility and X-ray photoemission measurements with new studies by the X-ray magnetic circular dichroism method. The observation of the Ce $M_{4,5}$ edges carried out with X-ray magnetic circular dichroism confirms the mixed-valence state deduced previously from X-ray photoemission spectroscopy. Moreover, we report on the measurements of the Ni $L_{2,3}$ edges, which enable verification of the Ni contribution to the magnetism of this compound.
\end{abstract}

PACS numbers: 71.20.Lp, 75.20.Hr, 78.70.Dm, 79.60.-i, 78.20.Ls

\section{Introduction}

Compounds based both on the lanthanides dominated by the $4 f$ levels and on the transition elements governed by the $3 d$ states are often a source of controversy if the magnetism of one of the elements tends to be damped [13]. The point is to decide if the magnetic properties of such compounds can be satisfactorily described by considering only either the $4 f$ or $3 d$ contribution. A good example of such a compound is $\mathrm{CeNi}_{4} \mathrm{~B}$ belonging to the series $\mathrm{RNi}_{4} \mathrm{~B}(\mathrm{R}=$ rare earth $)$. For this series of compounds it is usually assumed that the magnetism of nickel is negligible owing to the filling of the $3 d$ states. We have chosen the $\mathrm{CeNi}_{4} \mathrm{~B}$ compound to be studied in more details by the X-ray magnetic circular dichroism (XMCD) method, as it is a paramagnetic material.

From literature and our previous investigations $[2,4]$ we know that $\mathrm{CeNi}_{4} \mathrm{~B}$ is characterized by $\mu_{\mathrm{eff}}=$ $0.52 \mu_{\mathrm{B}} /$ f.u. and the paramagnetic Curie temperature $\theta=-10.7 \mathrm{~K}$, as obtained from the modified Curie-Weiss law, i.e. by including the temperature independent Pauli paramagnetism.

The X-ray photoemission spectroscopy (XPS) revealed a mixed-valence behavior of the Ce ions in the $\mathrm{CeNi}_{4} \mathrm{~B}$ compound. The occupation of the $f$-states $n_{f}=0.83$, which has been obtained from the analysis of the core Ce $3 d$ doublet [5] is in excellent agreement with the value derived by Mazumdar et al. [6] by the X-ray absorption near-edge structure (XANES) studies.

Both the literature and our previous studies do not finally decide about the role of the $\mathrm{Ni}$ atoms in the mag- netic properties of the $\mathrm{RNi}_{4} \mathrm{~B}$ compounds. Our theoretical calculations [1] for the ferromagnetic $\mathrm{GdNi}_{4} \mathrm{~B}$ compound using the spin-polarized tight binding linear muffin tin orbital (TB LMTO) method have indicated that the Ni magnetic moment is negligible $\left(\mu_{\mathrm{Ni}} \approx 0.018 \mu_{\mathrm{B}}\right)$ and is antiparallel to the rare-earth magnetic moment.

\section{Experimental}

The polycrystalline $\mathrm{CeNi}_{4} \mathrm{~B}$ compound was synthesized by the induction melting under an argon atmosphere. As the ingot the stoichiometric amounts of Ce, $\mathrm{Ni}$ and $\mathrm{B}$ were used. This compound crystallizes in the hexagonal $\mathrm{CeCo}_{4} \mathrm{~B}$ structure, which can be derived from $\mathrm{RNi}_{5}$ by replacing the two $\mathrm{Ni}$ atoms at the $2 \mathrm{c}$ sites in every second layer by $\mathrm{B}$ atoms. In the $\mathrm{CeCo}_{4} \mathrm{~B}$ structure the $\mathrm{Ni}$ atoms occupy $2 \mathrm{c}$ and $6 \mathrm{i}$ sites, the rare earth atoms are located in the 1a and $1 \mathrm{~b}$ sites, and the boron atoms occupy one position, $2 \mathrm{~d}$ [7]. The unit cell is illustrated in Fig. 1. The lattice parameters derived by fitting of the X-ray diffraction pattern with the program FullProf in the space group $P 6 / \mathrm{mmm}$ are $a=5.00 \AA$ and $c=6.99 \AA$.

The XMCD measurements were done on the high field magnet end station, at ID08, ESRF. The samples were filled with a diamond file under a base pressure of about $10^{-8}$ mbar and transferred immediately to the magnet for measurements at a pressure of about $5 \times 10^{-10}$ mbar. The spectra were obtained in the total electron yield (TEY) mode, i.e. the drain current in the sample was 


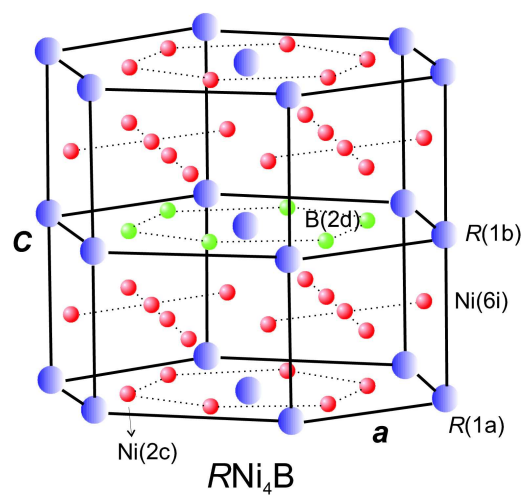

Fig. 1. Unit cell of the $\mathrm{RNi}_{4} \mathrm{~B}$ compounds. The lines are only a guide to the eye.

measured. The spectra are normalized by the incident beam intensity. The XMCD signal was determined by reversing both the ellipticity of the beam between circular left and right, and the applied magnetic field between $+4 \mathrm{~T}$ and $-4 \mathrm{~T}$.

\section{Results and discussion}

The starting point of the discussion is if one can expect a significant XMCD signal from cerium and, perhaps, from nickel in the case of the $\mathrm{CeNi}_{4} \mathrm{~B}$ compound. First information is provided by the magnetic susceptibility measurements. As it has been mentioned in the introduction, this compound appeared to be paramagnetic, at least down to $2 \mathrm{~K}$, and there has not been a wide linear region in the reciprocal magnetic susceptibility $\chi^{-1}(T)[2,4]$. Instead, $\chi(T)$ can be well fitted in the full temperature range using the modified Curie-Weiss law, $\chi(T)=\chi_{0}+C /(T-\theta)$, where $\chi_{0}$ is a temperature independent term and $\mu_{\text {eff }}=0.52 \mu_{\mathrm{B}} /$ f.u., $\theta=-10.7 \mathrm{~K}$. However, in the temperature range between $230-300 \mathrm{~K}$, $\chi^{-1}(T)$ is linear and can be fitted with the full effective magnetic moment $\mu_{\mathrm{eff}}=2.54 \mu_{\mathrm{B}} /$ f.u. and $\theta$ as large as $-955 \mathrm{~K}$. In the frames of the single ion Kondo model [8] this value of the paramagnetic Curie temperature implies a Kondo temperature $T_{\mathrm{K}}=|\theta / 2|=477 \mathrm{~K}$. Such a large value means that the Ce ions are in a mixed-valence state in the compound studied. The fully demonstrated effective magnetic moment of Ce means that the paramagnetic behavior can be described without considering the contribution of the $\mathrm{Ni}$ atoms.

The mixed valence state has been directly corroborated by the XPS measurements of the core Ce $3 d$ peaks [5]. Due to a finite probability of the excitation of the $f^{0}$ and $f^{2}$ states, leading to a different screening of the core hole, additional satellites can appear on the shoulders of the main Ce $3 d$ peaks. The analysis according to the Gunnarsson and Schönhammer model [9] provided the $f$-occupancy $n_{f}=0.83$ and the hybridization between the $f$ states and the conduction electrons $\Delta=85 \mathrm{meV}$, which implies a strong mixed valence behavior [5].
The X-ray absorption spectrum (XAS) measurement is one of the best methods to study the valence state. The high energy satellites visible in Fig. 2 at about $887.5 \mathrm{eV}$ and $905 \mathrm{eV}$ for the $\mathrm{Ce} M_{4,5}$ edges are characteristic of the mixed valent compounds with strong hybridization between the $f$ states and the conduction electrons $[6,10]$. The Ni $L_{2,3}$ edges for $\mathrm{CeNi}_{4} \mathrm{~B}$ are also displayed in Fig. 2.

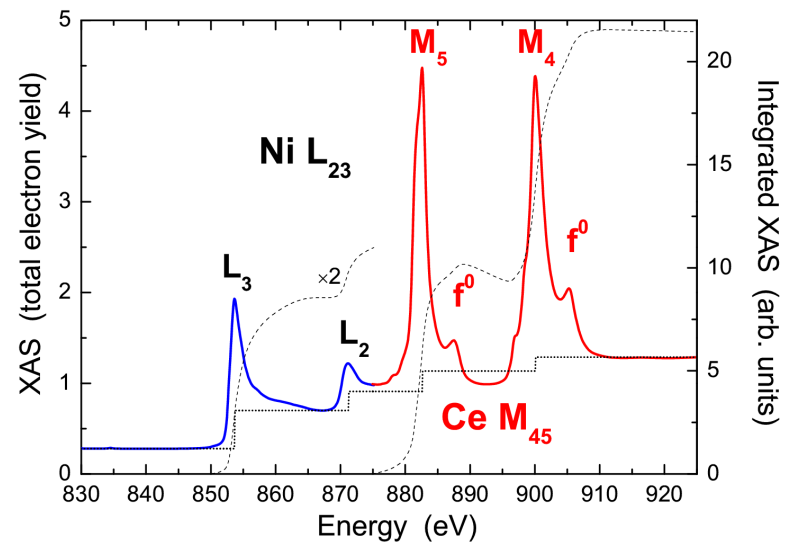

Fig. 2. XAS spectrum of the Ce $M_{4,5}$ and $\mathrm{Ni} L_{2,3}$ edges. The dotted line represents the step function describing the non-resonant absorption. The dashed line is the integral of the XMCD spectrum (right axes).

We have shown that the magnetic susceptibility, XPS and XAS measurements demonstrate consistently that $\mathrm{Ce}$ is in the intermediate valence state accompanied by a strong $4 f$-conduction electrons hybridization in $\mathrm{CeNi}_{4} \mathrm{~B}$. In spite of these observations the dichroic signal (Fig. 3), i.e. the difference between XAS of the two opposite beam polarizations, reveals that both Ce and Ni possess a small but measurable magnetic contribution. The shape of the spectra is in agreement with other Ce-based compounds [10] but an important observation concerns the mutual orientation of the $\mathrm{Ce}$ and $\mathrm{Ni}$ magnetic moments. It stems from Fig. 3 that these two contributions are antiparallel.

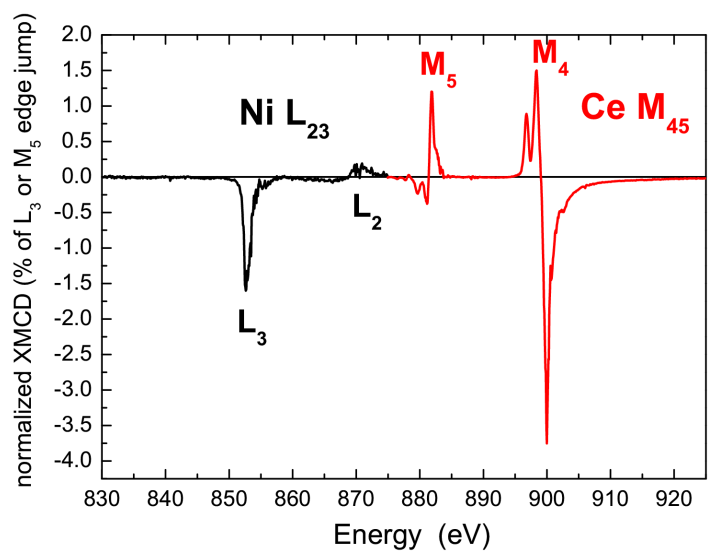

Fig. 3. XMCD signal for the Ce $M_{4,5}$ and $\mathrm{Ni} L_{2,3}$ edges. 
It is possible to estimate both the orbital and the spin contribution of the magnetic moment employing the standard analysis of the XAS and XMCD spectra [11, 12]. The orbital and the spin sum rules provide the mean value of the $z$ component of the orbital momentum operator

$$
\left\langle L_{z}\right\rangle=-\ln \frac{2 \int_{J^{+}+J^{-}}\left(I^{-1}-I^{1}\right) \mathrm{d} E}{3 \int_{J^{+}+J^{-}}\left(I^{1}+I^{-1}\right) \mathrm{d} E}
$$

and the average value of the $z$ component of the spin operator

$$
\begin{aligned}
& \left\langle S_{z}\right\rangle=-n_{h} \\
& \quad \times \frac{\int_{J^{+}}\left(I^{-1}-I^{1}\right) \mathrm{d} E-\frac{l}{l-1} \int_{J^{-}}\left(I^{-1}-I^{1}\right) \mathrm{d} E}{\int_{J^{+}+J^{-}}\left(I^{1}+I^{-1}\right) \mathrm{d} E},
\end{aligned}
$$

where $I^{-1}$ and $I^{1}$ denote the absorption coefficients for the right and left polarized photons. The integration is over the respective $J$ edges and $n_{h}$ corresponds to the number of holes in the $l$ shell. It is assumed that the magnetic dipole operator $\left\langle T_{z}\right\rangle$ is negligible, which is reasonable in the case of polycrystalline samples.

The continuum contribution (the non-resonant absorption) is introduced by a step function (Fig. 2) with steps height determined by the degeneracy of the core hole. The dashed line in Fig. 2 shows the integral of the $\mathrm{Ni} L_{2,3}$ and $\mathrm{Ce} M_{4,5}$ XAS (denominator in Eqs. (1) and $(2))$. The other integrals involved in the equations are obtained from the integration of the XMCD spectrum.

For $\mathrm{Ni}$ we assume $n_{h}=1.5$ (bulk value) and $l=2$. We get the orbital magnetic moment $m_{\mathrm{orb}}^{\mathrm{Ni}}=0.0039 \mu_{\mathrm{B}}$ and the spin magnetic moment $m_{\mathrm{spin}}^{\mathrm{Ni}}=2 \times\left\langle S_{z}\right\rangle=0.0087 \mu_{\mathrm{B}}$. These tiny values confirm the small contribution of $\mathrm{Ni}$ in magnetism of the $\mathrm{CeNi}_{4} \mathrm{~B}$ compound. One can also see that the spin and the orbital components are parallel. The ratio $m_{\mathrm{orb}}^{\mathrm{Ni}} / m_{\mathrm{spin}}^{\mathrm{Ni}}$ being 0.45 is in good agreement with the value of 0.46 found by Mizumaki et al. [13] for $\mathrm{GdNi}_{2}$ but significantly enhanced compared to Ni metal (0.095) [14].

For Ce we put $n_{h}=13.17$, based on the previous XPS studies [5] and $l=3$. We get the orbital magnetic moment $m_{\mathrm{orb}}^{\mathrm{Ce}}=0.161 \mu_{\mathrm{B}}$ and the spin magnetic moment $m_{\text {spin }}^{\mathrm{Ce}}=2 \times\left\langle S_{z}\right\rangle=-0.186 \mu_{\mathrm{B}}$. In the calculation of the spin component we have divided Eq. (2) by 1.6, which is a correction resulting from the off-diagonal term of the $3 d-4 f$ exchange interaction limiting the applicability of the spin sum rule for rare earths (for details see [15]).

The spin and the orbital components are antiparallel. The ratio $m_{\mathrm{orb}}^{\mathrm{Ce}} / m_{\mathrm{spin}}^{\mathrm{Ce}}$ is -0.86 . It is much smaller than -4 expected theoretically for $J=5 / 2$ results probably from the delocalization of the $4 f$ electron.

\section{Conclusions}

We have shown that the X-ray absorption measurements confirm the mixed valence state and strong
$4 f$-conduction electrons hybridization of the Ce ions in the $\mathrm{CeNi}_{4} \mathrm{~B}$ compound. Ni exhibits a very small but measurable magnetic signal in the X-ray magnetic circular dichroism. We have obtained $m_{\mathrm{orb}}^{\mathrm{Ni}}=0.0039 \mu_{\mathrm{B}}$ and $m_{\mathrm{spin}}^{\mathrm{Ni}}=0.0087 \mu_{\mathrm{B}}$. XMCD of the Ce $M_{4,5}$ edges have provided $m_{\mathrm{orb}}^{\mathrm{Ce}}=0.161 \mu_{\mathrm{B}}$ and $m_{\mathrm{spin}}^{\mathrm{Ce}}=-0.186 \mu_{\mathrm{B}}$. The XMCD spectrum indicates that the Ce and Ni magnetic moments are antiparallel.

\section{Acknowledgments}

This work was supported by the funds for science in years 2007-2009 as a research project (T. Toliński, A. Kowalczyk).

\section{References}

[1] T. Toliński, M. Pugaczowa-Michalska, G. Chełkowska, A. Szlaferek, A. Kowalczyk, Phys. Status Solidi $B$ 231, 446 (2002).

[2] N.M. Hong, N.P. Thuy, G. Schaudy, T. Holubar, G. Holscher, J.J.M. Franse, J. Appl. Phys. 73, 5698 (1993).

[3] P. Ravindran, S. Sankaralingam, R. Asokamani, Phys. Rev. B 52, 12921 (1995).

[4] T. Toliński, A. Kowalczyk, A. Szlaferek, M. Timko, J. Kováč, Solid State Commun. 122, 363 (2002).

[5] T. Toliński, A. Kowalczyk, M. Pugaczowa-Michalska, G. Chełkowska, J. Phys., Condens. Matter 15, 1397 (2003).

[6] C. Mazumdar, Z. Hu, G. Kaindl, Physica B 259-261, 89 (1999).

[7] Y.B. Kuz'ma, N.S. Bilonizhenko, Kristallografiya 18, 710 (1973).

[8] H.R. Krishnamurthy, K.G. Wilson, J.W. Wilkins, Phys. Rev. Lett. 35, 1101 (1975).

[9] O. Gunnarsson, K. Schönhammer, Phys. Rev. B 28, 4315 (1983)

[10] M. Finazzi, F.M.F. de Groot, A.-M. Dias, B. Kierren, F. Bertran, Ph. Sainctavit, J.-P. Kappler, O. Schulte, W. Felsch, G. Krill, Phys. Rev. Lett. 75, 4654 (1995).

[11] C.T. Chen, Y.U. Idzerda, H.-J. Lin, N.V. Smith, G. Meigs, E. Chaban, G.H. Ho, E. Pellegrin, F. Sette, Phys. Rev. Lett. 75, 152 (1995).

[12] E. Dartyge, F. Baudelet, C. Giorgetti, S. Odin, J. Alloys Comp. 275-277, 526 (1998).

[13] M. Mizumaki, K. Yano, I. Umehara, F. Ishikawa, K. Sato, A. Koizumi, N. Sakai, T. Muro, Phys. Rev. B 67, 132404 (2003).

[14] C.T. Chen, F. Sette, Y. Ma, S. Modesti, Phys. Rev. B 42, 7262 (1990).

[15] T. Jo, J. Electron Spectrosc. Relat. Phenom. 86, 73 (1997). 